Revue musicale OICRM

\title{
Formations pédagogiques musicales en Suisse. Des outils didactiques émergents
}

\author{
Isabelle Mili, Catherine Grivet Bonzon, Marianne Jacquin, Peter Knodt et Iris \\ Haefely
}

Volume 4, numéro 1, 2017

Apprendre et enseigner la musique au $\mathrm{XXI}^{\mathrm{e}}$ siècle. Nouvelles propositions pédagogiques

URI : https://id.erudit.org/iderudit/1040299ar

DOI : https://doi.org/10.7202/1040299ar

Aller au sommaire du numéro

Éditeur(s)

Observatoire interdisciplinaire de création et recherche en musique (OICRM)

ISSN

2368-7061 (numérique)

Découvrir la revue

Citer cet article

Mili, I., Grivet Bonzon, C., Jacquin, M., Knodt, P. \& Haefely, I. (2017). Formations pédagogiques musicales en Suisse. Des outils didactiques émergents. Revue musicale OICRM, 4(1), 44-66. https://doi.org/10.7202/1040299ar

\section{Résumé de l'article}

La présente recherche, soutenue par le Fonds national suisse (FNS), se consacre aux enseignements d'un groupe de didacticiens de la musique des Hautes écoles de musique (HEM) de Genève, Lausanne, Bâle, Lugano et Berne. Ces enseignements sont appréhendés comme susceptibles d'éclairer les changements intervenus dans la formation des formateurs et la nature de l'évolution des dispositifs. Les modes de professionnalisation des futurs enseignants de musique en fin de formation sont au coeur de cet article. Nous présentons, dans les quatre parties de cet article : les spécificités de cette recherche, y compris sa méthodologie mixte (quantitative et qualitative), l'analyse qualitative de modèles professionnels sous-jacents aux pratiques des formateurs, le corps dans une double fonction (comme outil pour enseigner et comme objet d'enseignement), les rôles et jeux de rôles inhérents aux variations didactiques d'un professionnel de l'enseignement. 


\title{
Formations pédagogiques musicales en Suisse. Des outils didactiques émergents
}

\author{
Isabelle Mili, Catherine Grivet Bonzon, \\ Marianne Jacquin, Peter Knodt et Iris Haefely
}

\begin{abstract}
Résumé
La présente recherche, soutenue par le Fonds national suisse (FNS), se consacre aux enseignements d'un groupe de didacticiens de la musique des Hautes écoles de musique (HEM) de Genève, Lausanne, Bâle, Lugano et Berne. Ces enseignements sont appréhendés comme susceptibles d'éclairer les changements intervenus dans la formation des formateurs et la nature de l'évolution des dispositifs. Les modes de professionnalisation des futurs enseignants de musique en fin de formation sont au cœur de cet article. Nous présentons, dans les quatre parties de cet article : les spécificités de cette recherche, y compris sa méthodologie mixte (quantitative et qualitative), l'analyse qualitative de modèles professionnels sousjacents aux pratiques des formateurs, le corps dans une double fonction (comme outil pour enseigner et comme objet d'enseignement), les rôles et jeux de rôles inhérents aux variations didactiques d'un professionnel de l'enseignement.

Mots clés : didactique ; enseignants ; formation ; musique ; professionnalisation.
\end{abstract}

\begin{abstract}
In this research, supported by the Swiss National Science Foundation (SNSF), the teaching practices of instrumental and vocal didacticians are analyzed from several points of view. At first, these teaching practices give us information about the evolution of forming devices that concern didactic professors in Higher Music Schools. But they also bring to light the type(s) of vocational training followed by the student teachers. In this paper, we present: the research methodology (quantitative and qualitative), the professional models that underpin the teaching practices, the double function of embodiment (as a tool to teach an instrument or to sing, as a subject to study), The variety of roles a didactician has to put on.
\end{abstract}

Keywords: didactics; music; professionalization; teachers; vocational training. 


\section{INTRODUCTION}

En mars 2016, la Haute École spécialisée de Suisse occidentale (HES-SO) publiait un projet d'école, schéma directeur, identifiant les forces les faiblesses et les pistes à suivre visant à sceller une convention d'objectifs ${ }^{1}$ pour la période 2015-2025. Si l'on prend en compte que la HES-SO constitue « une vaste université romande des métiers regroupant 19000 étudiants $^{2}$ ", cette convention d'objectifs ne peut manquer de mentionner quelques jalons caractéristiques de ces institutions tertiaires de formation et quelques outils dont celles-ci se dotent (militer en faveur des filières préprofessionnelles d'excellence, développer la dimension sociétale, mettre en œuvre et renforcer des axes de recherche, améliorer des locaux, etc.), y compris pour la formation aux métiers de l'éducation artistique. De fait, les Hautes écoles de musique (HEM Genève et HEMU Lausanne), ainsi que les Hautes écoles d'art et de design (HEAD Genève et ECAL Lausanne) qui en font partie, proposent des filières conduisant aux métiers de l'enseignement. Pour la HEM Genève, par exemple, 600 étudiants $^{3}$ en provenance de cinq continents peuvent notamment choisir une formation pédagogique conduisant au titre de Master of Arts in Pedagogy. Et cette " pédagogie " est explicitement située, du fait que l'institution s'affirme comme " issue du Conservatoire de musique de Genève " (fondé en 1835) " et de l'Institut Jaques-Dalcroze » (fondé en 1915)" En d'autres termes, l'institution de formation revendique à la fois :

- Un passé pédagogique ancestral, basé sur une approche institutionnalisée des savoirs (Martin-Balmori 2016), où la corporéité est construite au service du musical (Jaques-Dalcroze).

- Un passé proche qui marque une césure symbolique puisque la HES-SO n'est un établissement public autonome que depuis le $1^{\text {er }}$ avril 2014.

- Un présent avec des caractéristiques bien définies puisque la formation à l'enseignement instrumental et vocal est désormais l'affaire de professeurs de didactique également impliqués dans des projets de recherche et évoluant dans le système de Bologne (comme l'ensemble de leurs collègues des HEM de Suisse), le tout dans une filière de niveau universitaire - là où, il y a 15 ans, officiaient des professeurs de pédagogie ${ }^{5}$ nullement obligés de s'impliquer dans un système académique global.

- Un avenir, dans la mesure où « [l'institution] s'engage en faveur des valeurs éducatives et sociétales de la musique, aussi bien dans l'éducation générale que dans l'éducation spécialisée, préprofessionnelle et professionnelle ${ }^{6}{ }^{\prime}$. Les

\footnotetext{
1 Voir Haute école de musique de Genève 2016.

2 Voir https://www.hesge.ch/hem/lecole (consulté le 4 décembre 2016).

3 Le masculin englobe le féminin.

$4 \quad$ Haute école de musique de Genève 2016, p. 3.

5 Nous distinguons ici la pédagogie avec une centration plus forte sur la relation élève/professeur (modalités d'interaction avec l'élève, prise en compte de ses besoins ) de la didactique comme davantage liée au domaine, à la discipline et aux savoirs (méthodes de présentation des contenus, types de tâches, gestes didactiques).

$6 \quad$ Haute école de musique de Genève 2016, p. 7.
} 
professeurs de didactique instrumentale et vocale, qui assurent désormais la formation des futurs enseignants d'instruments et de chant, n'ont pas forcément eu l'occasion de dresser un bilan critique de la mutation de leur fonction. En témoigne le peu de recherches effectuées sur les aspects didactiques de la formation à l'enseignement instrumental et vocal (Bourg 2006, 2007 et 2008 ; Dubé et al. 2011 ; Dubé et Després 2012 ; Grivet Bonzon, Mili et Schumacher 2016 ; Jacquin et Grivet Bonzon 2017 ; Martin-Balmori 2016) depuis la grande réforme du système des Hautes écoles spécialisées (HES), une réforme qui a été amorcée en 1998 et achevée après 2010. Cette dernière décennie, en particulier, ne semble pas avoir offert beaucoup de possibilités d'analyse avec les outils des sciences de l'éducation.

La présente recherche, soutenue par le Fonds national suisse de la recherche scientifique (FNS) ${ }^{7}$, se consacre aux enseignements d'un groupe de didacticiens d'instrument ou de chant des HEM de Genève, Lausanne, Bâle, Lugano et Berne. Ces enseignements sont appréhendés comme susceptibles d'éclairer les changements dans la formation des formateurs et la nature de l'évolution des dispositifs. Dans une première partie, nous présentons les spécificités de cette recherche, y compris sa méthodologie mixte : quantitative et qualitative. Une deuxième partie est consacrée à l'analyse qualitative de modèles professionnels sous-jacents aux pratiques des formateurs. Au centre de notre troisième partie se trouve le corps comme outil pour enseigner et comme objet d'enseignement. Enfin, dans une quatrième et dernière partie, ce sont les rôles et jeux de rôles inhérents aux variations didactiques d'un professionnel de l'enseignement instrumental ou vocal qui focalisent notre attention.

\section{DÉCLINAISON DE LA PROBLÉMATIQUE ET SPÉCIFICITÉS DE LA RECHERCHE}

La recherche est organisée en deux grands groupes :

- Un premier qui s'est attaché aux indicateurs du processus de professionnalisation eux-mêmes.

- Un second qui s'est proposé de mettre sous la loupe les définitions données durant les cours de didactique instrumentale ou vocale - définitions portant sur les domaines de l'interprétation et de la technique, y compris les phénomènes qui relèvent de l'incorporation des savoirs.

Les thématiques (les modèles d'analyses et de professionnalisation, le rapport entre technique et interprétation, le fonctionnement des ressources sémiotiques - langagières et/ou corporelles, les rôles et jeux de rôles inhérents aux variations didactiques d'un professionnel de l'enseignement musical) sont toutes subordonnées à notre objet central : les logiques de professionnalisation. Mais nous n'avons pas postulé, a priori, un lien causal entre la formation et les pratiques des étudiants que nous avons observées.

Cette étude se focalise sur la question de l'organisation et de la conduite des enseignements à un double niveau (celui des 11 professeurs de didactique, comme

$7 \quad$ Projet de recherche FNS 100019_156730 : «La professionnalisation des enseignants en formation initiale. Analyses didactiques qualitatives et quantitatives des gestes professionnels en développement ». 
celui de leurs 20 étudiants). Les enseignements sont appréhendés sous l'angle d'une analyse de pratiques effectives et dans la perspective de leur finalité ultime, à savoir la production, chez des élèves d'école de musique, des apprentissages conduisant à une certaine maîtrise ou performativité. Le recueil de données porte sur :

- Une série de quatre leçons $\left(\mathrm{L}_{1}\right.$ à $\left.\mathrm{L}_{4}\right)$ données par un étudiant à un élève, sous la supervision du professeur de didactique concerné - avec une "leçon 0 » $\left(\mathrm{L}_{0}\right)$, qui les précède et qui sert de point de référence.

- Une série de rétroactions du professeur de didactique instrumentale ou vocale, en présence de tous ses étudiants (mais sans leur-s élève-s).

Partant du constat que la plupart des étudiants inscrits en Master of Arts in Pedagogy sont déjà titulaires ou en train de finaliser un master instrumental ou vocal, on peut considérer que l'étudiant en Master of Arts in Pedagogy se professionnalise de deux manières : soit il était musicien et devient enseignant, soit il doit simultanément démontrer qu'il est un musicien performant dans son domaine (musicien d'orchestre / membre d'un ensemble de musique de chambre / ou soliste) et un enseignant. Cela signifie que deux catégories de savoirs experts sont convoquées : les savoirs qui permettent d'affronter la scène et ceux qui permettent de conduire des leçons.

Le corpus de la recherche issu de 64 leçons filmées est constitué des verbatims (échanges oraux et interactions gestuelles) et des définitions apparaissant dans les cours de didactique (codées en double aveugle par des binômes constitués des membres de l'équipe $\mathrm{DAM}^{8}$, de 1'Université de Genève, et par des enseignants-chercheurs des HEM). L'analyse de ces verbatims et codages est réalisée dans une deuxième étape. Ce dispositif est centré essentiellement sur le cadre méthodologique de la recherche clinique en didactiques (Leutenegger 2000) qui se base sur l'observation, l'enregistrement vidéo et la transcription de leçons ou d'extraits de séquences.

Les études pilotes ou études de faisabilité sont fréquentes en recherche clinique. Une revue complète des principes guidant ces recherches peut être trouvée dans les travaux de Lancaster, Dod et Williamson (2004), Pinheiro et Bates (2000), Cochran et Cox (1992). Tous les gestes de définitions des professeurs de didactique et des étudiants en train d'enseigner à leur élève dans le cadre du cours de didactique ont fait l'objet d'une codification et d'un traitement statistique. Le but étant de bien distinguer corrélations et inférences causales, et d'expliquer certains effets (ou absence d'effets) en ayant recours à des modèles linéaires mixtes (MLM) et des modèles linéaires mixtes généralisés (GLMM).

Parmi les gestes qui permettent de caractériser les actions didactiques de l'enseignant en classe, nous avons choisi, pour l'ensemble de la recherche, d'étudier les trois gestes liés à la fonction de définition (de la situation, des tâches, des contenus d'enseignement). Nous avons identifié six types de définitions que l'on trouve potentiellement dans les cours de musique instrumentale et vocale : $\mathrm{D}_{\mathrm{T}}$ (définition tâche), $\mathrm{D}_{\mathrm{T}}$ (définition discursive), $\mathrm{D}_{\mathrm{E}+}$ (définition exemple), $\mathrm{D}_{\mathrm{E}-}$ (définition contre-exemple), $\mathrm{D}_{\mathrm{P}}$ (pointing définition ${ }^{9}$ ) et

8 Didactique des arts et du mouvement. Voir http://www.unige.ch/fapse/dam/ (consulté le 6 décembre 2016).

9 Définition ostensive à travers laquelle l'enseignant indique des éléments concernant les contenus d'enseignement. Il peut indiquer à travers des gestes, mais aussi verbalement (avec des déictiques). 
$\mathrm{D}_{\mathrm{M}}$ (définition métaphore). Afin de garder une perspective didactique liée aux contenus, nous avons également catégorisé quatre sortes de savoirs musicaux $(\mathrm{M})$ à enseigner : $\mathrm{M}_{\mathrm{T}}$ (savoirs techniques), $\mathrm{M}_{\mathrm{I}}$ (savoirs interprétatifs), $\mathrm{M}_{\mathrm{L}}$ (savoirs liés à la lecture de partitions), $\mathrm{M}_{\mathrm{H}}$ (savoirs liés au travail à la maison). Concernant les rétroactions des formateurs, nous avons aussi identifié trois sortes de savoirs didactiques $(\mathrm{D})$ pour enseigner : $\mathrm{D}_{\mathrm{L}}$ (processus d'apprentissage de la musique), $\mathrm{D}_{\mathrm{PL}}$ (planification) et $\mathrm{D}_{\mathrm{R}}$ (conduite de leçon).

Un travail de codage a été effectué à partir de verbatims des leçons données par les étudiants à un de leurs élèves, en présence du professeur de didactique, puis des rétroactions des professeurs à leurs étudiants, suite à l'observation de ces leçons. Ces codages ont été réalisés à partir d'observables variés, notamment les milieux didactiques (les tâches, les différents supports de l'enseignement), les échanges verbaux et les comportements des acteurs. Pour les leçons données par les étudiants à leurs élèves d'instrument ou de chant, les codages ont été réalisés dans le but de repérer les occurrences concernant les savoirs à enseigner ainsi que les types de définitions mobilisées pour leur enseignement/apprentissage. Concernant les séances de rétroactions, nous avons également codé des savoirs pour enseigner qui sont censés caractériser un discours de formation à la didactique de l'instrument ou du chant.

Les verbatims ont été codés selon la démarche représentée dans la figure 1.

\begin{tabular}{|c|c|c|c|c|c|}
\hline $\begin{array}{l}\text { Date du } \\
\text { cours : }\end{array}$ & $\begin{array}{l}\text { Professeur de } \\
\text { didactique : Th }\end{array}$ & $\begin{array}{l}\text { Acteurs : } \\
\text { Étudiant : Sth }\end{array}$ & Moments du cours : & $\begin{array}{l}\text { Codage } \\
\text { effectué } \\
\text { par: }\end{array}$ & $\begin{array}{l}\text { Référence } \\
\text { vidéo : }\end{array}$ \\
\hline Chrono & $\begin{array}{l}\text { Type(s) de } \\
\text { définition / type de } \\
\text { savoir / acteur }\end{array}$ & \multicolumn{2}{|c|}{$\begin{array}{l}\text { Verbatim ou mention du contenu qui fait } \\
\text { l'objet d'une définition }\end{array}$} & \multicolumn{2}{|c|}{$\begin{array}{l}\text { Savoir tacite } \\
\text { (ce que l'on vise à faire } \\
\text { apprendre) }\end{array}$} \\
\hline $01: 32$ & $\mathrm{D}_{\mathrm{R}} \mathrm{M}_{\mathrm{T}} \mathrm{Th}$ & \multicolumn{2}{|c|}{ 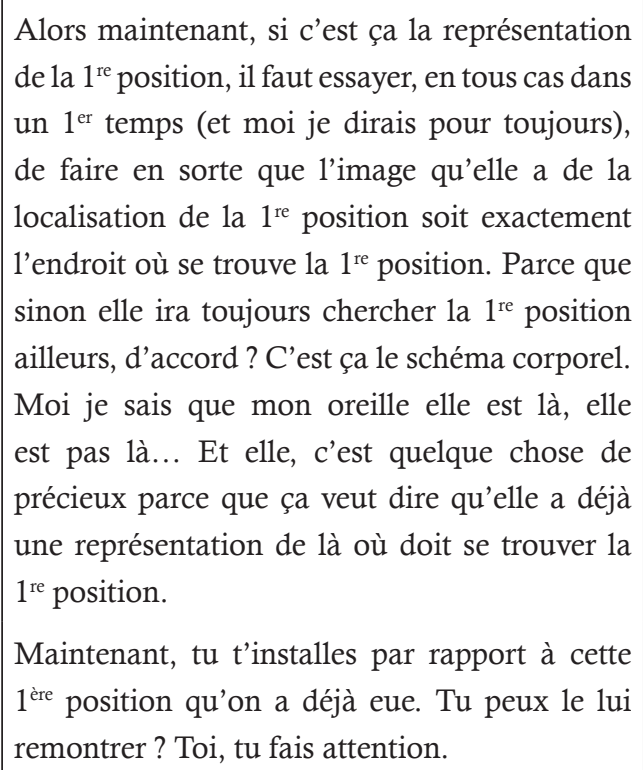 } & & \\
\hline $02: 40$ & $\mathrm{D}_{\mathrm{L}} \mathrm{M}_{\mathrm{T}} \mathrm{Th}$ & \multicolumn{2}{|c|}{$\begin{array}{l}\text { Voilà, ça c'est la posture. Parce que sinon ça ne } \\
\text { peut pas marcher si on fait des choses qui sont } \\
\text { contre la nature de l'élève. }\end{array}$} & & \\
\hline
\end{tabular}

Figure 1 : Exemple de codage (étalonnage minute de 00:00 à ...). 
RÔLE DES MODĖLES PROFESSIONNELS DANS LA FORMATION DIDACTIQUE D'UN ENSEIGNANT DE MUSIQUE BÂLOIS : LE CAS DE L'ENSEIGNEMENT DU TROMBONE

Nous visons à mettre en évidence les modèles professionnels sous-jacents aux pratiques des formateurs en observant dans les leçons données par les étudiants des traces de savoirs musicaux, didactiques et pédagogiques issus des séances de formation. Quels modèles rencontrés en formation sont repris par les étudiants ? Comment les étudiants se les approprient-ils ? Nous tentons de répondre à ces questions à partir d'une étude de cas portant sur la formation à l'enseignement du trombone d'un étudiant de la HEM de Bâle.

Cadre théorique : vers une définition opérationnelle des concepts de "modèle » et de "développement professionnel»

Traiter la question complexe des effets des modèles professionnels en formation sur le développement des pratiques enseignantes en musique présuppose la clarification d'au moins deux concepts : ceux de modèle et de développement professionnel.

Des savoirs font modèle lorsqu'ils font l'objet d'un partage possible au sein d'une communauté (de musiciens, d'enseignants, etc.) et lorsqu'ils sont rendus visibles en tant qu'objets de formation enseignés. Un modèle doit donc répondre aux critères suivants :

- Faire l'objet d'une conceptualisation et d'une systématisation.

- S'insérer dans un système de concepts ou de valeurs.

- Être thématisé, institutionnalisé et/ou modélisé en séance de formation.

Les formateurs disposent de toute une série de références comprenant, implicitement ou explicitement, des modèles sous-jacents. Parmi ceux-ci, nous retenons les six principes didactiques suivants : l'analogie partielle entre l'apprentissage de la musique et du langage (par exemple : Gordon 2001, Suzuki 1968) ; la représentation musicale qui précède la lecture des notes ; le développement des compétences musicales à l'aide des sensations du mouvement, de l'espace, du poids et de l'énergie ; la formation de la motricité fine découlant de la motricité globale ; l'apprentissage par le jeu - de l'instrument et par des activités ludiques (Mahlert 2011) ; l'articulation des savoirs musicaux techniques et de ceux portant sur l'interprétation des œuvres.

Sans pouvoir entrer ici en détail sur les contenus de ces six principes, nous faisons 1'hypothèse que ces modèles fonctionnent comme des outils du développement professionnel chez les étudiants. Nous considérons celui-ci sur un axe représentant un continuum entre deux pôles. Le premier pôle d'appropriation correspond à la capacité de reproduire un modèle. Par "reproduction " nous entendons une " appropriation spontanée de contenus, de normes d'action, de valeurs, de croyances, sans que cet ensemble soit pensé ni construit (Christoph Wulf [1999], cité dans Lemaître 2007, p. 13). Dans la pratique de l'étudiant, il s'agit d'une reprise mimétique d'un modèle rencontré en formation (par exemple, la reprise du discours du formateur, l'application d'un principe didactique). À l'autre extrême se situe le pôle que nous appellerons « reconfiguration", par analogie entre texte du savoir (Mercier 2002) et récit : «l'arrangement configurant transforme la succession des événements en une totalité signifiante qui est le corrélat d'assembler les événements et fait que l'histoire se laisse suivre. 
Grâce à cet acte réflexif, 1'histoire peut être traduite en une pensée " (Ricœur 1983, p. 130). Transposé dans l'enseignement, il s'agit d'une intégration des concepts comme outils de réflexion et d'une transformation des savoirs issus de la formation en savoirs professionnels (Vanhulle 2009, p. 249).

Sur la base de ces considérations théoriques, nous postulons qu'il y a développement professionnel lorsqu'il y a reprise et transformations observables des objets de formation suivants :

- Intégration pertinente d'un objet musical abordé en formation dans une leçon.

- Apparition de supports et d'outils didactiques rendus de plus en plus visibles pour l'élève.

- Emploi de gestes de définition, avec une augmentation de leur précision, de leur pertinence par rapport à l'objet enseigné et de l'articulation entre eux.

- Augmentation de l'explicitation et de l'étayage d'une tâche.

- Apparition des traces de conceptualisations dans le discours de l'étudiant.

\section{Méthodologie}

Cinq leçons (l'étudiant face à son élève) et cinq séances de formation (le formateur face à son étudiant) d'une durée moyenne de 20 minutes ont été filmées et ont d'abord fait l'objet d'une transcription partielle et d'un codage ${ }^{10}$. Comme nous nous intéressons aux gestes de définition, aux contenus enseignés et plus particulièrement aux traces des modèles issus de la formation, nous avons transcrit les consignes de travail, les pointages sur des contenus spécifiques (leur désignation), l'utilisation de métaphores et les explications. Nous avons cependant renoncé à une transcription exhaustive de toutes les nuances dans le discours de l'enseignant ou du formateur (ne figurent pas les hésitations, répétitions, intonations de la voix, etc.).

L'analyse qualitative s'est déroulée en trois temps. Nous avons d'abord élaboré un synopsis des leçons et séances de formation, afin de regrouper les activités dans une macrostructure rendant visibles à la fois la chronologie et la hiérarchie des principaux contenus présents dans la formation et l'enseignement (Schneuwly, Dolz et Ronveaux 2006). Ensuite, nous avons procédé à un premier repérage des contenus et gestes présents en formation et repris dans les leçons. À ce stade, nous avons émis les premières hypothèses sur les types de modèles sous-jacents aux contenus de la formation. Finalement, et sur la base des résultats issus de l'analyse quantitative et des indicateurs définis, nous avons sélectionné des passages significatifs du point de vue du développement professionnel et les avons analysés plus finement. L'analyse du discours des acteurs nous a fourni des indices, soit pour dégager des modèles (par exemple, la mention du mot "modèle " par le formateur ou des passages de récit d'expérience), soit pour repérer des traces de ces modèles dans les leçons (par exemple, la reprise des paroles du formateur ou la mention d'un outil proposé en formation). Le codage contextualisé des gestes de définition nous a en outre permis

10 Voir la section sur le rôle des modèles professionnels dans la formation didactique d'un enseignant de musique bâlois. 
de vérifier nos hypothèses quant à l'association des gestes de définition aux modèles repérés. Les codages ont notamment montré qu'au geste de définition discursive $\left(D_{D}\right)$ pouvait correspondre un modèle d'expertise musicale ou un savoir didactique issu de l'expérience du formateur - savoir institutionnalisé à un moment donné de la séance de formation.

\begin{tabular}{|c|c|}
\hline Le(s) modèle(s)... & Exemples : le formateur... \\
\hline ...philosophique, humaniste & $\begin{array}{l}\text {...évoque les finalités d'un enseignement de la } \\
\text { musique (le bonheur) et propose à son étudiant } \\
\text { d'aider son élève à oser s'exprimer à travers son } \\
\text { instrument. }\end{array}$ \\
\hline ...de l'expertise des grands musiciens & $\begin{array}{l}\text {...rappelle la tenue du violon, telle que Menuhin la } \\
\text { pratiquait. }\end{array}$ \\
\hline ...de l'enseignant/musicien engagé/impliqué & $\begin{array}{l}\text {...propose une tâche à l'étudiant, sous la forme d'un } \\
\text { concours, et lui propose de la reproduire avec son } \\
\text { élève. }\end{array}$ \\
\hline $\begin{array}{l}\text {...de l'enseignant/formateur chercheur, didacticien } \\
\text { créatif, expérimentateur }\end{array}$ & $\begin{array}{l}\text {...se définit lui-même comme constamment à la } \\
\text { recherche des raisons d'un obstacle chez l'élève ou } \\
\text { de moyens pour le dépasser. }\end{array}$ \\
\hline ...de l'enseignant/formateur réflexif & $\begin{array}{l}\text {... analyse une leçon avec l'étudiant et/ou modélise } \\
\text { la réflexion en intégrant le général (théories) au } \\
\text { particulier (cas observé en leçon). }\end{array}$ \\
\hline ...des savoirs d'expérience du formateur & $\begin{array}{l}\text {...décrit une pratique qui a des effets positifs et en } \\
\text { explique les raisons. }\end{array}$ \\
\hline $\begin{array}{l}\text {...issu(s) de la psychologie et pédagogie : } \\
\text { l'interaction avec l'élève }\end{array}$ & ...évoque l'importance de la notion de contact. \\
\hline $\begin{array}{l}\text {...issu(s) de la didactique : méthodes } \\
\text { d'enseignement, exercices, outils, gestes didactiques }\end{array}$ & $\begin{array}{l}\text {...présente des modèles d'organisation des contenus, } \\
\text { des aides pour l'apprentissage, des exercices, explicite } \\
\text { ou modélise des gestes à pratiquer pendant la leçon. }\end{array}$ \\
\hline ...normatif : la bonne leçon de musique & $\begin{array}{l}\text {....mentionne des critères de qualité d'une leçon (par } \\
\text { exemple, la variété des méthodes, le lien ente leçon et } \\
\text { travail à domicile, etc.) et les attentes liées à la leçon- } \\
\text { épreuve. }\end{array}$ \\
\hline
\end{tabular}

Figure 2 : Les modèles en formation.

\section{Résultats}

\section{Les modèles en formation}

Parmi les modèles de professionnalisation sous-jacents que nous avons mis au jour grâce à l'analyse de tout le corpus, nous avons pu repérer en particulier neuf catégories 
de modèles à partir de deux sous-ensembles de ce corpus (violon ${ }^{11}$ et trombone). Ces neuf catégories résultent d'une analyse thématique, basée sur des modèles existants (voir supra) et par regroupements successifs d'occurrences trouvées dans le corpus. Nous les présentons dans un classement (figure 2) allant du plus général au plus concret, en donnant un exemple de contexte dans lequel ils apparaissent. Chacun des modèles peut se manifester à deux niveaux : en tant que modèle présent dans le discours du formateur ou sous la forme d'une modélisation.

2. La traçabilité des modèles dans les leçons : l'importance des modèles didactiques et pédagogiques

Dans l'étude de cas du trombone, c'est avant tout la reprise des modèles didactiques (méthodes de présentation des contenus, types de tâches, gestes didactiques, etc.) et pédagogiques (modalités d'interaction avec l'élève, prise en compte de ses besoins, etc.) qui permet de mesurer le développement de l'étudiant. Six constats ressortent de nos analyses. Le premier porte sur le rapport étroit entre les objets musicaux travaillés en formation et dans les leçons données à un élève. L'étudiant reprend systématiquement soit des aspects techniques, soit un travail sur l'improvisation - qui ont fait l'objet d'une planification avec son formateur. Un deuxième constat porte sur l'augmentation de la variété des activités musicales proposées, des modalités et des outils pour l'élève. Alors que la leçon initiale $\left(\mathrm{L}_{0}\right)$ ne contient que deux activités (échauffement et jeu d'un morceau de blues), la deuxième leçon $\left(\mathrm{L}_{2}\right)$ comprend une phase de travail sur l'instrument en trois temps, dont chacun fournit le contexte pour travailler des savoirs musicaux différents (souffle, ornementations, accords, etc.) et sous des formes différentes (jeu ensemble, en solo, en alternance). En troisième, l'on constate un effet structurant de l'outil de planification sur les leçons de l'étudiant. Alors que la $\mathrm{L}_{0}$ consiste surtout en un jeu commun, dès la $\mathrm{L}_{2}$ apparaît une organisation des contenus selon une logique de progression. En quatrième, l'on observe l'intégration progressive de tâches à domicile dont les multiples fonctions ont été enseignées en formation. Les cinquième et sixième constats sont liés à la conception du formateur, à ce qu'implique pour lui l'acte d'enseigner : planifier sa leçon, s'engager dans la relation avec l'élève (jouer avec lui, lui donner une rétroaction constructive), lui donner des tâches précises et les moyens pour améliorer la qualité de son jeu (explications, verbalisations et exemples). La place plus importante que l'étudiant accorde à l'élève en $\mathrm{L}_{4}$ et le développement de la notion de contact témoignent de la reprise de ce modèle. Des progrès dans la précision des consignes, associées à des explications, sont également visibles. C'est ce dernier aspect que nous allons illustrer dans la partie suivante.

11 Pour l'enseignement du violon, nous disposons de quatre leçons d'une durée moyenne de 20 minutes et de sept séances de formation d'une durée moyenne de 60 minutes, mais nous ne traitons ici que des données concernant le trombone. 


\section{Illustration}

La figure 3 montre deux extraits. Le premier passage illustre une activité durant la phase d'échauffement en tout début de leçon $\left(\mathrm{L}_{0}\right)$. Le second extrait $\left(\mathrm{L}_{3}\right)$ se situe dans une leçon dont l'objet principal est de jouer le morceau de jazz Summertime. La comparaison entre les deux façons d'introduire et d'accompagner la tâche témoigne du développement de l'étudiant.

\begin{tabular}{|c|}
\hline $\mathbf{L}_{0}$ \\
\hline $\begin{array}{l}\text { J'aimerais maintenant jouer quelques sons ensemble, si tu en as envie. Nous pouvons faire des exercices de } \\
\text { glissandi. Je joue et ensuite tu répètes }[\ldots . . \text {. Ou bien nous pouvons jouer ça ensemble... peut-être que nous } \\
\text { pouvons continuer à exercer ça... Tu peux choisir une des deux lignes et faire un rythme... par exemple... ou } \\
\text { la ligne. }\end{array}$ \\
\hline $\mathbf{L}_{3}$ \\
\hline $\begin{array}{l}\text { J'aimerais, si tu en as envie, que tu fasses des ornementations sur les deux premières mesures [...]. } \\
\text { Tu peux simplement faire des ornementations sur la première voix, sur les deux premières mesures, faire une } \\
\text { ornementation, peu importe quoi. Oui alors ici, c'est la ligne de basse... La première mesure reste en fa majeur } \\
\text { et ensuite le } 5^{\mathrm{e}} \text { niveau donc } 5 \text { et ensuite } 6,5,6[\ldots] \text {. Tu dois montrer un peu plus cette } d o, d o^{7}\left(l^{\prime} e n s e i g n a n t ~ j o u e\right) \\
{[\ldots] \text {. Ça [les ornementations] aide pour gagner en liberté, pour obtenir le style et connaître les accords [...]. }} \\
\text { Peux-tu revenir sur la deuxième mesure et jouer maintenant en solo? Et, simplement, c'est le même sentiment, } \\
\text { tu sais maintenant ce qui se passe dans la basse et ça t'aide }[\ldots] \text {. Tu dois penser ainsi, comme un son long (il } \\
\text { joue) et ça bouge }[\ldots] \text {. C'est simplement deux noires, une fois do, une fois ré, mais avec une ornementation }[\ldots] \text {. } \\
\text { Je crois que si tu joues les noires brièvement, ça ira mieux. }\end{array}$ \\
\hline
\end{tabular}

Figure 3 : Évolution dans la formulation des consignes et l'accompagnement dans la tâche.

L'évolution est d'abord clairement visible dans la manière de donner les consignes. Celles-ci sont très sommaires en $\mathrm{L}_{0}$, énoncées selon le mode de la proposition ( "j'aimerais jouer ", " nous pouvons ", " tu peux choisir ») et trahissant une certaine indécision de la part de l'étudiant ("peut-être », « ou »), elles deviennent plus précises en $\mathrm{L}_{3}$ par une délimitation de l'extrait du morceau sur lequel porte l'exercice (« les deux premières mesures ", « la tonalité »), les explications qui l'accompagnent quant à la tonalité ( $f a$ majeur »), le rythme ( deux noires ») et une posture plus directive (" tu dois montrer ", "tu dois penser »). Ensuite, l'objet technique (les glissandi) travaillé en $\mathrm{L}_{0} \mathrm{n}$ 'a aucun rapport avec une œuvre, alors qu'en $\mathrm{L}_{3}$ les ornementations sont à exécuter en référence à une œuvre musicale. Ainsi, les différents objets techniques (accords, rythme, etc.) sont mis en relation entre eux et au service de l'improvisation (les ornementations). Finalement, en $\mathrm{L}_{3}$, l'exécution de la tâche est accompagnée par deux dimensions absentes en $\mathrm{L}_{0}$ : la mention de la finalité de l'exercice (« ça aide pour ", "si tu joues ainsi, ça ira mieux ») et la construction d'une mémoire expérientielle (" c'est le même sentiment ", " tu sais maintenant ce qui se passe dans la basse et ça t'aide").

Des cinq critères de transformation définis plus haut, quatre sont présents dans cet exemple. Premièrement, l'étudiant travaille une œuvre musicale abordée en formation à laquelle il subordonne les aspects techniques. Ces derniers ne sont pas travaillés comme une fin en soi, mais au service du jeu interprétatif. Deuxièmement, les outils 
proposés à l'élève pour lui faciliter l'entrée dans l'œuvre musicale sont nommés, leurs fonctions sont explicitées. Troisièmement, le geste de définition de la tâche, devenu plus explicite, est associé à des notions dont a besoin l'élève afin de mieux se rendre compte des gestes nécessaires à son jeu. Quatrièmement, la tâche est étayée, l'étudiant guide l'élève en lui fournissant progressivement les outils nécessaires pour améliorer son jeu. S'appuyant sur des modèles de la formation, l'agir de l'étudiant devient ainsi à son tour modèle pour son élève, dans la mesure où il thématise les savoirs et que ses gestes entrent en relation, formant de la sorte un système cohérent qui permet de rendre les objets accessibles et de leur donner du sens.

LE CORPS, OUTIL POUR ENSEIGNER ET OBJET D'ENSEIGNEMENT AU CENTRE DU PROCESSUS DE FORMATION D'UN ENSEIGNANT EN VIOLONCELLE

Le corps, un enjeu de la professionnalisation des professeurs de musique

Alors qu'ils ont été investigués par les chercheurs pour l'enseignement primaire et secondaire (Jorro et Crocé-Spinelli 2010 ; Pujade-Renaud 1983 et 2005), les savoirs professionnels liés à la corporéité au sein des organismes de formation professionnalisante de l'enseignement de la musique constituent un champ de recherche encore en grande partie à parcourir ${ }^{12}$.

Partant du principe que nous recherchons des indices de l'évolution des étudiants en master de pédagogie de la musique, nous tentons de mettre à jour une évolution du geste didactique et de la transformation des modalités mises en place par les étudiants dans le dispositif didactique ; cela en particulier dans l'organisation et la conduite des enseignements par les étudiants, à travers la mise en scène de leur propre corps et du corps de l'élève comme outils de compréhension, de progrès et de performativité instrumentale de l'élève, que celle-ci soit de l'ordre de la technique ou de l'interprétatif.

\section{Cadre théorique}

En prenant comme point de départ l'étude de la perception, Merleau-Ponty (1945) fait $\mathrm{du}$ " corps propre », la condition permanente de l'expérience humaine comme ouverture perceptive au monde. Pour le philosophe, le primat de la perception induit le primat de l'expérience, dans la mesure où la perception revêt une dimension active. Connaître son corps, inscrit dans un faisceau d'habitudes, dans une relation à la culture dans laquelle il est baigné, c'est savoir s'en servir. C'est ce corps qui fait que le violoncelle n'est pas la même chose pour l'élève débutant ou pour quelqu'un qui ne joue pas de violoncelle que pour le violoncelliste expert, qui en naturalise l'usage. Le défi principal de l'enseignant en formation est bien de déconstruire cette naturalisation -obstacle à la transposition didactique, pour procéder à une objectivation des savoirs professionnels.

12 Voir à ce propos les travaux de Mili (2014), Martin-Balmori (2016), Hoppenot (1981), Desroches, Stévance et Lacasse (2014). 
Nous assistons à une primeur du corps sur l'émergence du sens et enracinons notre réflexion dans une double dimension : celle du corps communiquant dans le rapport didactique enseignant/élève et celle du corps des protagonistes comme lieu de l'expérience et d'incarnation des savoirs. L'expérience, liée au corps musicien, est à la fois celle de l'élève et celle du professeur dans la situation de formation ; car c'est bien de cela dont il s'agit ici : d'une corporéité au centre du jeu didactique, en référence à la théorie de l'action conjointe en didactique (Sensevy, Mercier et Schubauer-Léoni 2000) et du dispositif de formation en alternance de deux HEM de Suisse romande (Merhan, Ronveaux et Vanhulle 2007).

L'étude prend sens dans la relation mise en place à un double niveau de transaction entre professeur de didactique/stagiaire, d'une part, et stagiaire/élève, d'autre part (Gagnon 2010). Elle s'appuie sur la théorie vygotskienne qui, bien que réservant une place fondamentale aux pairs et à l'environnement socioculturel de l'apprenant, désigne néanmoins l'enseignant médiateur comme un acteur majeur dans la transmission du savoir (Demerval et White 1993). Nous nous prononçons aussi pour une appropriation des concepts vygotskiens dans le cadre de la formation professionnelle à condition de prendre en compte la spécificité des rapports entre apprentissage et développement à l'âge adulte.

\section{Méthodologie}

S'appuyant sur la méthodologie générale de l'étude ${ }^{13}$, ce paragraphe porte spécifiquement sur l'observation de cinq leçons données par un étudiant $\left(\mathrm{L}_{0}\right.$ à $\left.\mathrm{L}_{4}\right)$ et quatre rétroactions de son professeur $\left(\mathrm{F}_{1}\right.$ à $\left.\mathrm{F}_{4}\right)$. Après avoir codé les gestes d'enseignement de chaque synopsis (figure 1) ainsi que les types de contenus enseignés, nous avons extrait des " moments remarquables " - remarquables pour le professeur qui les thématise comme pour le chercheur qui les analyse. Ces moments possèdent un potentiel heuristique significatif, lié à la corporéité, à un recours au corps dans sa double dimension matérielle et symbolique, comme " outil pour transmettre " et " objet de savoir ». Une observation fine des indices de professionnalisation s'est de plus ancrée dans le décryptage des séquences filmées, à travers les mouvements et postures de l'étudiant dans la relation à l'élève.

\section{Résultats}

L'analyse du corpus à travers les indicateurs retenus révèle que si l'expérience des corps est bien un objet d'apprentissage, le primat de la corporéité est aussi un enjeu de formation, dans le sens où celle-ci est investie de fonctions multiples, tant au regard du corps de l'élève (souvent élémentarisé à des fins de performances techniques ou instrumentales) qu'au regard de celui de l'enseignant. À cet étudiant-enseignant reviennent des fonctions plurielles : celles du corps qui pointe, qui montre l'exemple, qui corrige les postures, qui joue en duo, mais qui fait parfois obstacle aux apprentis-

13 Voir la section sur la déclinaison de la problématique et les spécificités de cette recherche. 
sages incorporés de l'élève. En effet, si l'étudiant investit progressivement les potentialités du corps comme outils et l'espace « terrain de jeu » de la transmission (nombre et longueur des déplacements et recours à la corporéité effectués lors de chaque séance, adéquation entre le geste de définition et le recours corporel comme outil), le corps reste l'objet d'enseignement des techniques et des processus interprétatifs dans des actions majoritairement distinctes les unes des autres et réparties dans le temps.

\section{La dimension spatiale de l'expérience des corps}

Issu à la fois de la forme scolaire (Vincent 1994) et des spécificités liées à l'instrument lui-même, l'espace scolarisé d'enseignement de la musique s'inscrit dans une tradition révélatrice des espaces axiologiques de l'institution formatrice autant que des besoins des acteurs. Ainsi, l'espace ne peut se réduire au simple rôle de contexte de l'activité, il offre au contraire un pôle dynamique de relations dans l'appropriation que s'en font les acteurs (Grivet Bonzon 2011). Dans le cours de violoncelle, l'élève et le stagiaire sont assis, formant un angle de 70 degrés, un jeu en miroir. Il y a deux chaises, deux violoncelles, un pupitre pour la partition de l'élève et aucun moyen de diffusion musical dont on pourrait faire usage. L'enseignant stagiaire est le maître de la mobilité de l'élève dont il régule la position instrumentale et l'espace de travail autour d'un axe unique, le violoncelle. Il dispose à la fois des objets et des corps qu'il organise, tant dans le souci d'une efficacité technique que de la bonne posture - celle qui ne blessera pas l'élève. Le stagiaire a une conception plutôt statique de son rôle, relevée par son formateur à plusieurs reprises - ce dernier le stimulant dans l'appropriation de l'espace à sa disposition ainsi que dans sa réflexion sur l'utilisation du corps comme objet de savoir pour enseigner.

$\mathrm{F}_{1}$ : « Maintenant, je me suis dit que je ne te trouvais pas assez exigeant pour tout ce qui concerne l'archet (geste) et l'aspect déplacement spatial de l'archet (geste) . Parce que de lui dire "prends tout l'archet ", c'est une chose, et de lui faire visualiser le talon (geste) quand on a presque l'index qui est contre la corde (geste) puis la pointe... c'est vraiment là (geste). Et lui faire observer géographiquement parlant (geste) comment ça se passe. Tu le fais pas assez. Et du point de vue du constat de comment je me tiens au talon et comment est la pointe, lui faire observer comment est son épaule (geste), son coude (geste) ; ça fait qu'il a une représentation un peu trop floue en fait de ce que c'est que de prendre tout l'archet ou un demi archet (geste) ; et ça, tu pourrais plus clarifier ».

Il est à noter que si les premières leçons offrent peu d'observations de déplacements, la routine du déroulement du cours prévalant sur l'espace de liberté potentiel, l'étudiant intègre au fur et à mesure de la formation les déplacements dans la palette sémiotique de ses gestes professionnels. L'action de l'étudiant observé reste cependant en deçà des attentes du professeur, qui éprouve le besoin de les réitérer de manière appuyée jusqu'en fin de formation.

$\mathrm{F}_{3}$ : «Deux remarques... Fais semblant de jouer du violoncelle (il se lève). Imaginons, je sais pas, que je trouve que tu as le coude gauche trop bas. 
Une façon de faire c'est ça (il bouge sèchement le coude de l'étudiante) et une autre façon de faire c'est ça (il bouge coude et épaule, de manière plus douce). Tu vois? La deuxième est un peu plus sympa. Quand tu le touches, tu l'attaques! Quand on veut amener un élève à faire un geste, il faut l'accompagner, sinon (geste) tu le secoues un peu et c'est pas comme ça qu'on développe la sensation du geste. Il va se dire OK je suis trop haut alors (geste) « chting », je suis trop bas (geste); mais si tu le modules avec lui en fait corporellement, il enregistre la sensation. Tout comme tu vois quelqu'un de ta famille qui est triste, tu vas le câliner (geste de câlin). Comme ça, plus entourant, plus chaleureux ».

\section{Le corps comme objet d'enseignement des techniques}

Les choix professionnels des enseignants sont déterminés par leur propre rapport au savoir et par la culture de l'institution. La complexité fait partie de la situation didactique dans le sens où les enseignants usent constamment de leur corps pour enseigner et ce la plupart du temps de manière inconsciente (Pujade-Renaud 2005). Chez le musicien débutant, l'apprentissage de techniques du corps met en évidence la prise de conscience de quelque chose d'étranger à soi, la distance entre soi et le soi que le contexte normatif lié à la culture de l'instrument l'enjoint à devenir pour rejoindre " l'idéaltype » disciplinaire ou pour aborder l'œuvre. À ce titre, c'est d'abord pour et par les apprentissages et les savoirs techniques que le corps est mis à contribution lors des leçons.

La structure triadique du cours (échauffement / exercices / travail sur une ouvre), dans sa récurrence systématique lors de toutes les leçons données par l'étudiant, pose la question du cadre de référence en vigueur dans l'institution de formation autant que celle de la fidélité à un modèle traditionnel d'enseignement de la musique basé sur le découplage entre exercices techniques et travail interprétatif. Les tâches dévolues à l'élève dans une première partie du cours (20 minutes pour un cours de 30 minutes) sont principalement centrées sur des savoirs techniques.

Ces éléments sont liés, entre autres :

- À la tenue de l'instrument, à la posture : les attitudes et postures participent non seulement aux prémices de l'activité, mais sont réactivées tout au long de la leçon, et ce dans chacune d'elles. Condition sine qua non d'une intégration $\mathrm{du}$ geste instrumental, tenir son instrument est aussi la condition d'une santé pérenne du corps. Autrement dit, tenir son instrument est un savoir à enseigner propice à une meilleure performativité ainsi qu'à l'expression de la musicalité. Installer l'élève, placer l'instrument sont parmi les gestes incontournables de l'activité enseignante du stagiaire. Si la tenue de l'instrument est référencée culturellement, le formateur souligne la prise en compte de la morphologie et de l'observation de l'élève et y consacre de longs développements lors des rétroactions.

- À la détente corporelle et à l'équilibre comme préalables au jeu : le juste milieu entre détente et tonicité est une des composantes problématiques pour le jeune enseignant face à l'élève débutant. Les interventions de l'étudiant tendent à une prise de conscience de l'importance de la détente musculaire, 
à des fins d'habileté technique et d'une corporéité globale de type postural, avec la respiration comme source de concentration dans l'anticipation du jeu et l'intériorisation du tempo.

On observe une décontextualisation et une désyncrétisation des savoirs musicaux lors des tâches effectuées sous forme d'exercices. Le corps y est fragmenté, les gestes sont élémentarisés avant de demander à l'élève de " penser un peu plus global ». Une fois les tâches et exercices corporels et techniques transformant le corps de l'élève en corps instrumentiste et les automatismes acquis par l'exercice et la répétition, il est postulé que l'élève pourra, dans une seconde phase, être en mesure d'interpréter. Lors des rétroactions, le professeur invite l'étudiant à prendre conscience du rapport entre l'espace investi et la technique au service de l'interprétation.

$\mathrm{F}_{3}$ : «C'est un peu philosophique, le rapport espace-temps. C'est pareil en musique - en tous cas avec les archets -, mais je pense qu'on peut décliner sur autre chose. C'est parfois l'espace qu'on utilise qui donne le temps et ce n'est pas le temps qui contraint l'espace».

\section{La sensation au service de l'interprétation}

Mili (2014) nous rappelle que les acquisitions techniques sont d'autant plus significatives pour l'élève qu'elles ne sont pas isolées de la notion d'interprétation. Nous l'avons vu précédemment, le temps consacré aux apprentissages interprétatifs par les étudiants est généralement limité, l'œuvre n'apparaissant la plupart du temps que dans le dernier tiers de la leçon. C'est souvent à partir de ce dernier tiers qu'entrent en scène des contenus d'enseignements liés à l'interprétation ainsi que le recours au corps pour la recherche de la " musicalité ». Les gestes d'enseignement de l'étudiant oscillent entre les tâches liées aux ressentis de l'élève et celles liées à un outillage corrélé aux moyens de la traduction musicale de l'émotion.

Lors de la leçon, l'imitation du geste instrumental et le jeu en duo impliquent le corps des agents, dans une corporéité interprétative. Si apprendre sans comprendre est décrié à juste titre par l'ensemble des didactiques, l'enseignement de l'instrument s'appuie très souvent sur l'exemple incontournable du " maître ». Le mimétisme du geste juste, de la posture adéquate reste à la base de l'enseignement duel tel qu'il est majoritairement pratiqué dans les institutions d'enseignement spécialisé de la musique. Outil fondamental de l'arsenal didactique dans les disciplines musicales, il est un point de repère pour l'élève - qui le transformera pour peu que l'enseignant lui en laisse la possibilité. Un des indices de l'usage de l'imitation lors des leçons est le recours aux exemples, articulés aux phases de définition de la tâche pour l'élève. La dévolution qui s'y enracine, loin d'être un acte vide de sens, s'inscrit dans la dynamique corporelle du professeur qui s'engage par l'exemple, physiquement et artistiquement. Le recours à l'accompagnement de l'élève et du jeu en duo, signe d'un engagement physique de l'étudiant stagiaire, est aussi largement investi parmi les milieux mis en place. 
RÔLES ET JEUX DE RÔLES INHÉRENTS AUX VARIATIONS DIDACTIQUES D'UN PROFESSIONNEL DE L'ENSEIGNEMENT MUSICAL

Pas d'enseignement sans jeu de rôle(s)! La théorie didactique mentionne explicitement la topogenèse comme composante essentielle de l'action didactique (Sensevy et Mercier 2007). Dans les institutions de formation musicale, les rôles ont peut-être légèrement "bougé ", au sens où la référence à un maître, très caractéristique des filiations d'interprètes, s'est doublée d'une référence à un ensemble de méthodes pédagogiques dépassant très largement les modes de transmission vécus par les futurs professeurs en tant qu'élèves. Appelé à dépasser la reproduction pédagogique de ce qu'il a lui-même dans sa formation instrumentale ou vocale, l'étudiant est invité à moduler ses interventions.

L'objectif de cette partie de notre texte est de mettre en évidence les traces d'un jeu de rôle différencié - traces telles qu'elles apparaissent dans notre recueil de données - et les relations de ce jeu de rôle avec l'apprentissage autonome de l'élève, ainsi que de suivre le développement de la variation didactique de l'étudiant. Par conséquent, nous focalisons notre attention sur les trois sous-questions suivantes :

- Peut-on observer, à partir de la quantité et la diversité des verbes d'action dans le discours, des changements qualitatifs dans l'agir didactique de l'étudiant?

- Quels sont les indices d'un jeu de rôle différencié du côté de l'étudiant?

- Y a-t-il des indices d'une relation entre le jeu de rôle et l'apprentissage autonome de l'élève?

Le point de départ pour la recherche d'indices de professionnalisation est une analyse partielle des résultats issus des codages effectués lors de la première phase de recherche.

\section{Cadre théorique}

Outre l'action conjointe en didactique (Sensevy et Mercier 2007), les processus de modification de l'action et de l'interprétation grâce à un jeu de rôle ont été traités par Mead (1934), Goffmann (1998) ou encore Dreitzel (1970). La prise de rôle (role taking), la négociation et l'interprétation des rôles par les acteurs ainsi que les processus de socialisation peuvent nous aider à nous orienter dans les situations de formations professionnelles comme celle qui est analysée ici. Les situations de rétroaction, en particulier, caractéristiques du suivi des étudiants, constituent un terrain très riche pour observer les actions qui relèvent de l'évolution des rôles. Le discours, mais aussi ce qui n'est pas dit, les mimiques, les gestes et les éléments d'une communication multimodale (Gebauer 2012) ayant fait l'objet de transcriptions, constituent la base de nos analyses.

Analyse

L'analyse partielle des résultats de codage, des leçons $\mathrm{L}_{0}$ à $\mathrm{L}_{3}$ de l'étudiant et de son élève - distantes de cinq mois - montre une augmentation moyenne d'un coefficient de 1.34 des gestes de définition de l'étudiant. Les augmentations des contenus portent en particulier sur les devoirs ( $2 \mathrm{x})$, l'interprétation et la technique (1.34 $\mathrm{x}$ chacune) ainsi que les sous-catégories " pointing » (désignation d'un élément précis) $(2.5 \mathrm{x})$ et tâche (nature exacte de la tâche à accomplir) $(1.625 \mathrm{x})$, ce qui correspond à une progression 
remarquable. Dans la microanalyse de la leçon $\mathrm{L}_{3}$, on se trouve confronté, durant 1 min $58 \mathrm{~s}$, à une densité très particulière, que nous estimons devoir être examinée de plus près. Ensuite, nous comparons cet extrait de $\mathrm{L}_{3}$ avec un extrait de la leçon $\mathrm{L}_{0}$.

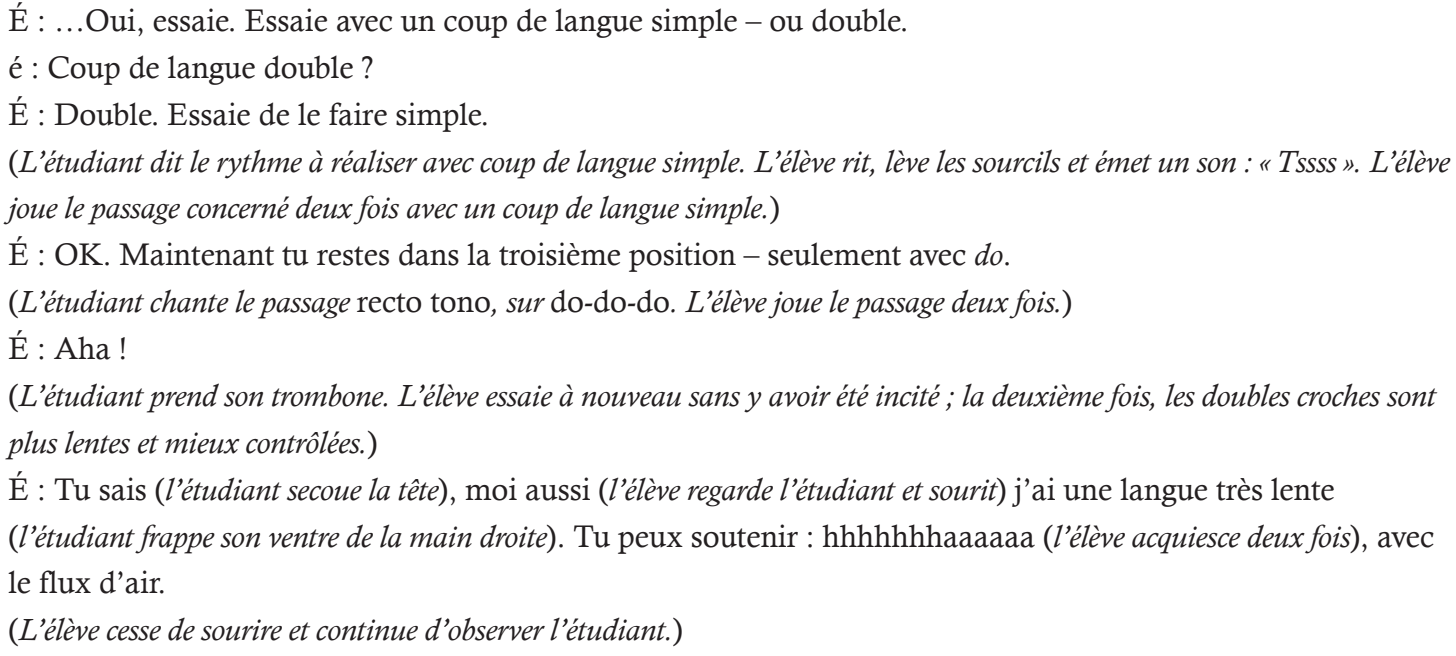

Figure 4 : Extrait de transcription en leçon $L_{3}(E ́=$ étudiant ; é = élève $)$.

Nous notons l'alternance d'essais successifs de l'élève et de conseils donnés par l'étudiant. Ces conseils reposent sur des définitions techniques (coup de langue simple ou double) et sur des définitions des tâches visant à jouer un passage difficile avec succès. L'élément remarquable du côté de l'élève est sa prise d'initiatives : il répète spontanément l'expérience à laquelle l'étudiant le convie. Du côté de l'étudiant, l'élément remarquable est son positionnement topologique : après avoir simplifié la tâche (renoncer provisoirement au coup de langue double), il se met « dans la position de l'apprenant » en exprimant une difficulté anatomique avec emphase (" moi aussi j'ai une langue très lente").

Afin de mieux cerner ce positionnement topologique dans son évolution, nous allons recenser les verbes d'action utilisés par l'étudiant durant les leçons $\mathrm{L}_{0}$ et $\mathrm{L}_{3}$. En $\mathrm{L}_{3}$, ce sont 52 verbes différents qui apparaissent dans son discours, contre 14 en $\mathrm{L}_{0}$; ce qui représente une progression de coefficient 2.6.

\begin{tabular}{|l|l|}
\hline Verbes d'action de $\mathbf{L}_{3}$ & $\begin{array}{l}\text { Différenciation des rôles de } \\
\text { l'enseignant (par 1'étudiant) }\end{array}$ \\
\hline Conduire, gesticuler, abandonner, convier, réguler, évaluer, pronostiquer & Maître \\
\hline Changer, diriger, jouer un exemple, montrer comment faire & Musicien/instrumentiste \\
\hline Élémentariser, montrer, indiquer, claquer (doigts ou langue) & Instructeur \\
\hline $\begin{array}{l}\text { Regarder, suivre, écouter, encourager (donner un feedback), expirer (de } \\
\text { l'air), s'adapter, acquiescer, approuver }\end{array}$ & Partenaire \\
\hline Expliciter, questionner, observer, laisser agir, argumenter & Architecte \\
\hline S'autoévaluer, révéler, manifester & Connaisseur de soi-même \\
\hline Essayer, exercer, répéter, créer & Apprenant \\
\hline
\end{tabular}

Figure 5: Verbes d'action de la leçon $L_{3}$ et rôles de l'étudiant (en tant qu'enseignant débutant). 
Les indices pour une modification des rôles de l'étudiant (dans le cas particulier : enseignant de trombone en devenir) sont particulièrement lisibles dans trois moments clés extraits de la leçon $\mathrm{L}_{3}$ :

a) "J'aimerais travailler avec toi le passage depuis $C$ ».

b) «Simplement, nous allons maintenant jouer des petits éléments - la première mesure ».

c) «Tu sais [l'étudiant secoue la tête], moi aussi [l'élève regarde l'étudiant et sourit] j'ai une langue très lente ».

D'après la théorie de l'action conjointe en didactique, il convient maintenant de se tourner vers l'élève pour trouver des indices qui montrent qu'une plus grande variation des actions de l'étudiant favorise l'action de l'élève. Nous allons l'examiner sous deux aspects : l'apprentissage autonome et l'interaction dialogique.

Les sept indicateurs pour l'apprentissage autonome (Weinert 1982) de l'élève peuvent être décrits selon l'auteur comme suit :

a) L'élève commence à jouer immédiatement après l'exemple donné.

b) L'élève répète un exercice sans demander une aide supplémentaire.

c) Après l'exercice, l'élève garde son regard dirigé sur le pupitre (et ne cherche pas l'approbation du professeur du regard).

d) Malgré la rétroaction de l'étudiant, l'élève continue de s'exercer.

e) L'élève autoévalue sa performance (de manière discursive ou non verbale, avec une mimique, ou avec le corps).

f) Les résultats de l'exercice de l'élève se transforment continuellement (sont en voie d'amélioration).

g) À l'issue de ses essais successifs, l'élève ne reçoit qu'une rétroaction partielle de l'étudiant ou des directives (modèles/gestes), en rapport à l'évolution de ses performances.

Les trois indicateurs pour l'interaction dialogique (Schmid 2014) avec l'élève peuvent être décrits comme suit :

a) À une question de l'étudiant, la réponse de l'élève est directe, avec un contenu clair et un regard en direction de l'étudiant. Il y a correspondance entre ce qu'il dit et son attitude corporelle.

b) La manière dont l'étudiant se manifeste : direction du regard et " habitus corporel ", apparaît comme révélatrice et extrêmement concise à la fois.

c) La rétroaction est adaptée à la situation et témoigne d'une attente positive quant aux performances qui vont suivre.

\section{Résultats}

L'augmentation de coefficient 2.6 dans la variété des actions didactiques, mesurée à l'aide des verbes d'action, s'appuie à la fois sur une variation du champ lexical (verbes) et sur une variation des rôles endossés par l'étudiant dans sa dernière phase de professionnalisation en formation initiale. Quant à la variation des rôles proprement dite, elle apparaît comme une suite de " je " complémentaires les uns des autres, façonnée de manière créative et qui composent un rôle professionnel socialement polymorphe. 


\begin{tabular}{|l|l|l|l|l|l|l|}
\cline { 2 - 7 } \multicolumn{1}{c|}{} & $\mathrm{D}_{\mathrm{T}}$ & $\mathrm{M}_{\mathrm{H}}$ & $\mathrm{D}_{\mathrm{P}}$ & $\mathrm{D}_{\mathrm{D}}$ & $\mathrm{D}_{\mathrm{M}}$ & $\mathrm{D}_{\mathrm{E}+}$ \\
\hline $\begin{array}{l}\mathrm{L}_{0} / 22.01 .2015 \\
\text { Durée }: 18: 30\end{array}$ & 8 & 1 & 0 & 6 & 1 & 7 \\
\hline $\begin{array}{l}\mathrm{L}_{3} / 11.06 .2015 \\
\text { Durée }: 29: 57\end{array}$ & 21 & 3 & 4 & 8 & 2 & 10 \\
\hline
\end{tabular}

Figure 6: Résultats.

Les sept indicateurs d'un apprentissage autonome peuvent être reliés à quatre composantes : 1'auto-observation, la définition des objectifs, le choix des stratégies et la rétroaction dans un modèle d'entraînement cyclique. Par conséquent, les conditions d'une interaction entre ces quatre composantes peuvent ou non être remplies. Dans le cas qui nous occupe, les indices pour un apprentissage autonome ont du moins pu être dégagés et nous incitent à affirmer que la variation des rôles et des actions de l'étudiant vont dans le sens d'une professionnalisation avérée.

Enfin, les actions observées chez l'élève et qui sont caractérisées par des traits typiques des actions autonomes doivent être mises en relation avec la densité des actions des deux protagonistes de l'enseignement musical : l'élève et l'étudiant, dans le contexte de ces leçons. Or cette densité plaide, avec la différenciation des rôles de l'étudiant, pour la mise en place, au fur et à mesure des leçons, d'un mode d'action conjointe où le guidage par l'étudiant et la prise en charge des apprentissages par l'élève arrivent à coexister.

\section{CONCLUSION}

Les résultats nous amènent à penser que les outils employés dans la construction du processus de professionnalisation ont trait à la fois au rapport fort et souvent inégal entre technique et interprétation, à la construction d'un corps spécialisé, à la prégnance des modèles d'apprentissage sous-jacents et à l'expérimentation d'une palette de rôles. Quant au rapport fort et souvent inégal entre ce que l'on pourrait nommer la technique et l'interprétation, dans la didactisation du rapport à l'œuvre comme milieu didactique (Brousseau 1990), nous constatons la tendance, chez les enseignants débutants, à privilégier des savoirs de type technique instrumentale, génériques et plutôt décontextualisés. Tout se passe comme si ces derniers constituaient la base, la condition au jeu instrumental, reléguant le rapport aux œuvres dans une fonction seconde.

En d'autres termes, le rapport aux œuvres, comme " levier » de l'apprentissage instrumental ou vocal, est quasiment inexploité. L'apprentissage d'un instrument de musique, notamment à ses débuts, implique de la part de l'enseignant qu'il procède à un découplage des contenus relatifs à la maîtrise du rapport corporel à l'instrument et des contenus relatifs à la musique qui peut être produite avec cet instrument (répertoire, modes de jeu, improvisation). Bien sûr, le découplage entre contenus orientés vers le technique et contenus orientés vers l'interprétatif découle d'un besoin qui est voué à être dépassé, dans la mesure où tout enseignant espère que ses élèves instrumentistes puissent jouer des œuvres et jouer de la musique. Cependant, les effets de cette nécessité 
didactique peuvent être fort variés, en ce qui concerne l'organisation de l'enseignement (contenus et types d'activités d'apprentissage). Se pose alors une question centrale : celle des réarticulations visant le jeu musical en tant qu'une totalité dans laquelle ces deux aspects (technique et interprétation) s'articulent organiquement. Il s'agit davantage de se référer à ce découpage comme nous aidant à instaurer des repères qui permettent de qualifier les tendances ou orientations des activités didactiques proposées aux élèves débutants dans le cadre des leçons d'instruments de musique ou du chant. Ainsi, par exemple, ce n'est pas parce que, dans une leçon, l'enseignant aborde une pièce musicale comme objet principal, que l'activité est orientée par des contenus relatifs à l'interprétation musicale. Dans le même ordre d'idées, un travail sur un aspect considéré comme essentiellement technique (par exemple le piqué des cordes sur le manche du violon ou du violoncelle), comme moyen de produire un son à la "bonne hauteur", peut être aussi orienté par l'enseignant vers des attentes esthétiques liées à la construction d'un «beau son ».

La construction d'un corps techniquement spécialisé et extrêmement sollicité est un élément dominant lors des interactions didactiques. L'importance du corps dans les différents modèles de formation musicale se révèle primordiale, dans l'appropriation tant du corps pour enseigner que du corps comme garant des savoirs à enseigner. L'étudiant se retrouve dans l'obligation de gérer, d'une part, le rapport au corps de l'élève au service des apprentissages (que ceux-ci soient d'ordre technique ou interprétatif), et, d'autre part, le rapport intime à son propre corps - qu'il didactise comme ressource et comme vecteur de l'enseignement.

Quant à la prégnance des modèles d'apprentissage sous-jacents à tous ces éléments, concernant les processus de professionnalisation en jeu, nous pensons que tous les modèles méritent d'être analysés - non pas tant pour leur " efficacité " (qu'il faudrait définir et qui nécessiterait une autre approche), mais pour leur potentiel d'enrichissement des outils didactiques au service de la formation à l'enseignement de la musique. C'est la dimension formative de ces modèles qui nous intéresse en dernière analyse et non pas leur réputation en termes de résultats "probants".

Enfin, l'expérimentation d'une palette de rôles vise à faire croître la part d'autonomie de l'élève. Expérimentation qui, comme l'ensemble des outils inhérents au processus de professionnalisation, est étroitement liée aux interactions entre l'étudiant et son formateur.

BIBLIOGRAPHIE

Abbott, Andrew D. (1988), The System of Professions. An Essay on the Division of Expert Labor, Chicago, The University of Chicago Press.

Altet, Marguerite (2004), «L'analyse de pratiques en formation initiale des enseignants. Développer une pratique réflexive sur et pour l'action ", Éducation permanente, n 160 , p. 101-110.

Association Européenne des Conservatoires (2010), Instrumental- und Gesangslehrerausbildung. Europäische Perspektiven. Polifonia-Arbeitsgruppe für Instrumental- und Gesangslehrerausbildung, http://www.aec-music.eu/userfiles/File/customfiles/aec-handbook-instrumental-undgesangslehrerausbildung-europaische-perspektiven-de- 20160229135446.pdf, consulté le 6 décembre 2016. 
Bourg, Adrien (2006), " Analyse comparative des notions de transposition didactique et de pratiques sociales de référence. Le choix d'un modèle en didactique de la musique? », Journal de Recherche en Éducation Musicale, vol. 5, $\mathrm{n}^{\circ}$ 1, p. 79-116.

Bourg, Adrien (2007), " Didactique du piano. Phénomènes de transposition autour d'une œuvre extraite du Petit livre d'Anna Magdalena Bach », dans Danièle Pistone (dir.), Pianos \& pianistes dans la France d'aujourd'hui, Paris, Université de Paris-Sorbonne / Observatoire musical fançais, p. 101112.

Bourg, Adrien (2008), «Didactique de la musique. Apports d'une approche comparatiste ", Éducation et didactique, vol 2, $\mathrm{n}^{\circ} 1$, p. 69-88.

Brousseau, Guy (1990), «Le contrat didactique. Le milieu », Recherches en didactique des mathématiques, vol. 9, no 3, p. 309-336, http://guy-brousseau.com/wp-content/uploads/2012/02/contrat-milieu1. pdf, consulté le 6 décembre 2016.

Cochran, William G., et Gertrude M. Cox (1992), Experimental Designs, 2e édition, New York, John Wiley \& Sons.

Demerval, René, et Florence White (1993), «La psychologie de Vygotsky et la pédagogie de la situation problème ", Spirale. Revue de recherches en éducation, no 10-11, p. 37-48, https://spirale-edu-revue.fr/ IMG/pdf/3 Demerval.pdf, consulté le 6 décembre 2016.

Desroches, Monique, Stévance, Sophie, et Serge Lacasse (dir.) (2014), Quand la musique prend corps, Montréal, Presses de l’Université de Montréal.

Dreitzel, Hans P. (1970), Patterns of Communicative Behavior, New York, Macmillan.

Dubé, Francis, et al. (2011), "Représentations d'attitudes posturales saines de professeurs de piano enseignant à des élèves du précollégial. Étude exploratoire ", Les Cahiers de la Société québécoise de la recherche en musique, vol. 12, nº 1-2, p. 103-133.

Dubé, Francis, et Jean-Philippe Després (2012), « Proposition d'un cadre conceptuel pour aider le professeur d'instrument à intégrer l'improvisation musicale à son acte pédagogique ", Intersections. Revue canadienne de musique, vol. 32, nº 1-2, p. 143-166.

Gagnon, Roxane (2010), Former à enseigner l'argumentation orale. De l'objet de formation à l'objet enseigné en classe de culture générale, Thèse de doctorat, Université de Genève, https://archive-ouverte.unige. ch/unige:6777, consulté le 6 décembre 2016.

Gebauer, Heike (2012), «"Wir beginnen mit unserem normalen Warm-Up...”, Inszenierungsmuster kognitiver Aktivierung im Musikunterricht - ein Fallbeispiel aus einer Videostudie ", dans Horst Bayrhuber et al. (dir.), Formate Fachdidaktischer Forschung. Empirische Projekte - historische Analysen - theoretische Grundlegungen, Münster, Waxman, p. 219-236.

Goffmann, Erving (1998), Les rites d'interaction, Paris, Minuit.

Gordon, Edwin E. (2001), Preparatory Audiation, Audiation and Musical Learning Theory. A Handbook of a Comprehensive Music Learning Sequence, Chicago, GIA Publications.

Grivet Bonzon, Catherine (2011), «Enjeux sociaux, culturels et éducatifs de la pratique autonome des musiques amplifiées chez les adolescent», Thèse de doctorat, Université Lumière Lyon 2.

Grivet Bonzon, Catherine, Mili, Isabelle, et Jérôme Schumacher (2016), « Des filières d'enseignement musical "de qualité", pour encourager "les talents" ? ", Revue suisse de sciences de l'éducation, vol. 38, $\mathrm{n}^{\circ} 3$, p. 495-512.

Haute école de musique de Genève (2016), Projet d'école 2015-2025, https://www.hesge.ch/hem/ sites/default/files/contributions/160309 projetecolehem-geneve web.pdf, consulté le 4 décembre 2016.

Hoppenot, Dominique (1981), Le violon intérieur, Paris, Van de Velde.

Jacquin, Marianne, et Catherine Grivet Bonzon (2017 [à paraître]), " Définir des tâches scolaires. Regards croisés entre cours de musique et cours de langue ", Actes colloque ARCD (Recherches comparatistes en didactique), Toulouse, Jean-Jaurès.

Jorro, Anne (2002), Professionnaliser le métier d'enseignant, Paris, ESF. 
Jorro, Anne, et Hélène Crocé-Spinelli (2010), « Le développement de gestes professionnels en classe de français. Le cas de situations de lecture interprétative ", Pratiques. Linguistique, littérature, didactique, p. 125-140, https://pratiques.revues.org/1527, consulté le 6 décembre 2016.

Lancaster, Gillian A., Dodd, Susanna, et Paula Williamson (2004), « Design and Analysis of Pilot Studies. Recommandations for Good Practice », Journal of Evaluation in Clinical Practice, vol. 10, $\mathrm{n}^{\circ} 2$, p. $307-312$.

Lefeuvre, Gwénaël, Garcia, Audrey, et Ludmila Namolovan (2009), «Les indicateurs de développement professionnel », Questions vives, vol. 5, n 11, http://questionsvives.revues.org/627, consulté le 22 juin 2016.

Lemaître, Denis (2007), « Entre savoirs et identités. Le phénomène de mimesis dans la formation des ingénieurs ", Les Sciences de l'éducation. Pour l'Ère nouvelle, vol. 3, no 40, p. 11-29, https://www. cairn.info/revue-les-sciences-de-1-education-pour-1-ere-nouvelle-2007-3-page-11.htm, consulté le 6 décembre 2016.

Leutenegger, Francia (2000), "Construction d'une "clinique" pour le didactique. Une étude des phénomènes temporels de l'enseignement ", Recherches en didactique des mathématiques, vol. 20, $\mathrm{n}^{\circ} 2$, p. 209-250.

Mahlert, Ulrich (2011), Wege zum Musizieren. Methoden im Instrumental- und Vokalunterricht, Mainz, Schott Verlag.

Martin-Balmori, Isabel (2016), « Didactique du belcanto : approche épistémologique des contenus d'enseignement et des pratiques de transmission ", Thèse de doctorat, Université de Genève, https://archive-ouverte.unige.ch/unige:88094, consulté le 6 décembre 2016.

Mead, George H. (1934), Mind, Self, and Society from the Standpoint of a Social Behaviorist, édité et introduit par Charles W. Morris, Chicago, University of Chicago Press, https://brocku.ca/MeadProject/ Mead/pubs2/mindself/Mead 1934 toc.html, consulté le 6 décembre 2016.

Mercier, Alain (2002), "La transposition des objets d'enseignement et la définition de l'espace didactique, en mathématiques ", Revue française de pédagogie, n 141, p. 135-171.

Merhan, France, Ronveaux, Christophe, et Sabine Vanhulle (2007), Alternances en formation, Bruxelles, De Boeck.

Merleau-Ponty, Maurice(1945), Phénoménologie dela perception, Paris, Gallimard, http://classiques.uqac. $\mathrm{ca} /$ classiques/merleau ponty maurice/phonomenologie de la perception/phonomenologie de la perception.pdf, consulté le 6 décembre 2016.

Mili, Isabelle (2014), L'œuvre musicale entre orchestre et école. Une approche didactique de pratiques d'écoute musicale, Berne, Peter Lang.

Pinheiro, José, et Douglas M. Bates (2000), Mixed-Effects Models in S and S-PLUS, Berlin, Springer Verlag.

Pujade-Renaud, Claude (1983), Le corps de l'enseignant dans la classe, Paris, ESF.

Pujade-Renaud, Claude (2005), Le corps de l'élève dans la classe, Paris, L'Harmattan.

Ricœur, Paul, (1983), Temps et récit, vol. 1, Paris, Seuil.

Schneuwly, Bernard, Dolz, Joaquim, et Christophe Ronveaux (2006), " Le synopsis. Un outil pour analyser les objets enseignés ", dans Marie-Jeanne Perrin-Glorian et Yves Reuter (dir.), Les méthodes de recherche en didactiques, Villeneuve d'Ascq, Septentrion, p. 175-189.

Schmid, Silke (2014), Dimensionen des Musikerlebens von Kindern. Theoretische und empirische Studie im Rahmen eines Opernvermittlungsprojektes, Augsburg, Wißner.

Sensevy, Gérard, et Alain Mercier (2007). Agir ensemble. L'action didactique conjointe du professeur et des élèves, Rennes, Presses universitaires de Rennes.

Sensevy, Gérard, Mercier, Alain, et Maria Luisa Schubauer-Léoni (2000), «Vers un modèle de l'action didactique du professeur à propos de la course à 20 ", Recherches en didactique des mathématiques, vol. 20, $n^{\circ}$ 3, p. 263-304, https://www.unige.ch/fapse/gredic/files/3314/1924/5082/Sensevy 2000 Modele action didactique professeur course a 20.pdf, consulté le 6 décembre 2016. 
Suzuki, Shinichi (1968), Nurtured by Love. A New Approach to Talent Education, Miami, Florida, Warner Bros. Publication.

Vanhulle, Sabine (2009), « Dire les savoirs professionnels. Savoirs de référence et logiques d'action », dans Rita Hofstetter et Bernard Schneuwly (dir.), Savoirs en (trans)formation. Au cour de l'enseignement et de la formation, Bruxelles, De Boeck Supérieur, p. 245-263, https://www.cairn.info/savoirs-entrans-formation--9782804115104.htm, consulté le 6 décembre 2016.

Vincent, Guy (dir.) (1994), L'éducation prisonnière de la forme scolaire? Scolarisation et socialisation dans les sociétés industrielles, Lyon, Presses universitaires de Lyon.

Vygotski, Lev (1985), Pensée et langage, Paris, Messidor.

Weinert, Franz E. (1982), "Selbstgesteuertes Lernen als Voraussetzung, Methode und Ziel des Unterrichts », Unterrichtswissenschaft, vol. 10, nº 2, p. 99-110. 